This item was submitted to Loughborough's Research Repository by the author.

Items in Figshare are protected by copyright, with all rights reserved, unless otherwise indicated.

\title{
Performance of variable rate and variable power MQAM system based on bit error rate and channel estimates
}

PLEASE CITE THE PUBLISHED VERSION

\section{PUBLISHER}

(C) IEEE

\section{VERSION}

VoR (Version of Record)

\section{LICENCE}

CC BY-NC-ND 4.0

\section{REPOSITORY RECORD}

Ong, Lay Teen, Mohammad Shikh-Bahaei, Jonathon Chambers, and Sangarapillai Lambotharan. 2019. "Performance of Variable Rate and Variable Power MQAM System Based on Bit Error Rate and Channel Estimates". figshare. https://hdl.handle.net/2134/5616. 
This item was submitted to Loughborough's Institutional Repository (https://dspace.lboro.ac.uk/) by the author and is made available under the following Creative Commons Licence conditions.

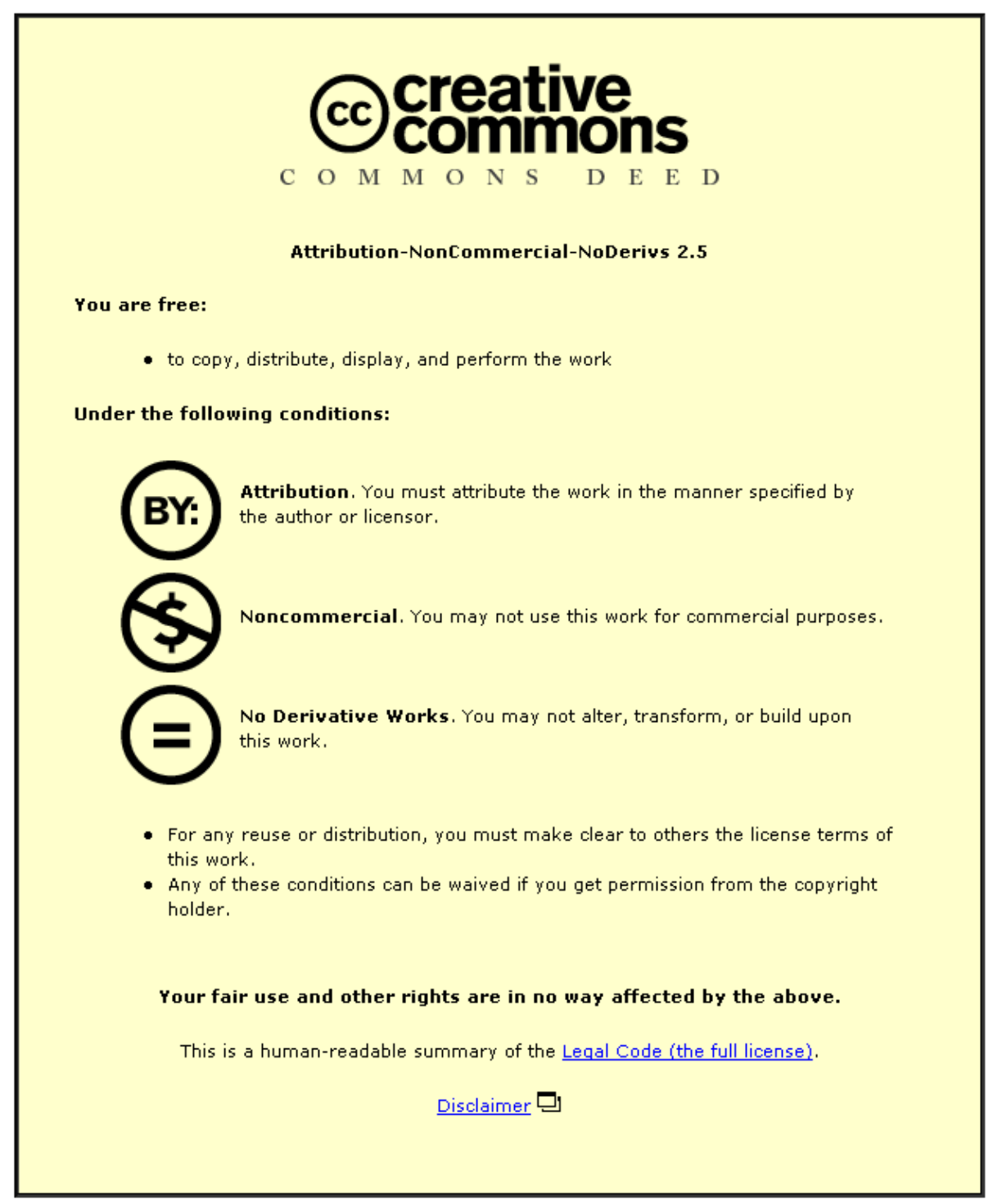

For the full text of this licence, please go to: http://creativecommons.org/licenses/by-nc-nd/2.5/ 


\title{
Performance of Variable Rate and Variable Power MQAM System Based on Bit Error Rate and Channel Estimates
}

\author{
L.T. Ong *, M. Shikh-Bahaei ${ }^{+}$, J.A. Chambers *, S. Lambotharan* \\ *Centre of Digital Signal Processing, Cardiff University, Cardiff CF24 OYF, UK \\ Email: OngL@cardiff.ac.uk \\ 'Department of Electronic Engineering, King's College London, London WC2R 2LS, UK \\ Email: m.sbahaei@kcl.ac.uk
}

\begin{abstract}
We consider the effect of an imperfect channel estimate on a variable rate and variable power (VRVP) multilevel quadrature amplitude modulation (MQAM) system, where the transmit power and data rate are varied relative to the estimate of the received signal. Based on the VRVPMQAM system operating over a Rayleigh flat-fading channel, we derive a bit error rate (BER) estimator using a maximum a posteriori (MAP) estimation approach. We apply the BER estimator to the VRVP-MQAM system, and thereby derive and obtain system expressions which are applicable to any channel estimation technique. In addition, the resulting formulations are also useful for analyzing the effect of channel state information (CSI). Consequently, we derive and obtain an optimal solution for our proposed system and generate numerical results to compare the variations of system performance with imperfect CSI.
\end{abstract}

\section{INTRODUCTION}

Robust and spectrally efficient communication over fading channels is a prerequisite for high-speed wireless data transmission. One common approach is through link adaptation, where the transmitter adapts its power, data rate, and coding scheme to match the channel variation using channel state information (CSI) available at the transmitter and/ or receiver. The capacity of a single-user channel with adaptive rate and power control has been examined in [1] and [2]. In [3], a variable rate variable power MQAM modulation technique which maximizes spectral efficiency over a fading channel was derived. One important premise for any adaptive transmission technique is the availability of accurate knowledge of CSI. Imperfect CSI, such as inaccurate channel estimates at the receiver and delayed estimate feedback to the transmitter are common parameters that may degrade the performance of any system incorporating adaptive techniques. Several papers have addressed these issues: [3] analyzed the effect of delay and estimation error in instantaneous signal-to-noise ratio (SNR) on bit error rate (BER), [4] analyzed and showed the effect of several system parameters, considering an instantaneous SNR estimate based on the minimum mean-square error (MMSE) criterion.

In this paper, we analyze the performance of rate and power adaptation based on estimates of instantaneous BER and SNR over a Rayleigh flat-fading channel. We proposed a system model incorporating a BER estimator derived using the MAP estimation method. Based on the proposed model, we derive and obtain analytical expressions for optimal rate and power adaptation to maximize spectral efficiency while satisfying an average power constraint and BER target. In relation to [3][4], our derivation for the power and rate control algorithm incorporates the correlation coefficient of the true channel gain and its estimate. Therefore our proposed algorithm provides a useful study on the impact of CSI, independent of a particular channel estimation technique used.

The remainder of this paper is organized as follows. In section II we describe the system model and analysis. We present our analytical approach to determining the BER estimate model in section III. Using these results, we next derive analytical expressions for optimum rate and power adaptation to maximize spectral efficiency in section IV. In section $\mathrm{V}$, we show numerical results to support the performance of our proposed system. We further show the impact of an imperfect channel estimate upon short-term variations on spectral efficiency and average power. Finally, conclusions are drawn in section VI.

\section{System Model ANd ANALysis}

Fig. 1 shows the system model for our analysis. We represent the channel model in discrete-time with statistically stationary and ergodic time-varying gain $\alpha(i)$ and additive, zero mean, white Gaussian noise $n(i)$, with spectral density $N_{0} / 2$. We consider pilot symbol assisted modulation (PSAM) [5] for the fading estimation technique with channel 


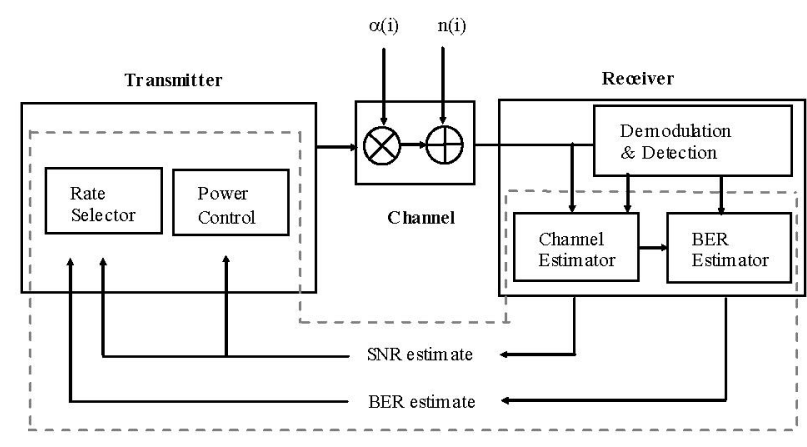

Figure 1. System Model.

gain estimates $\hat{\alpha}(i)$ obtained from the pilot symbols recovered at the receiver. For a constant transmit power $\bar{S}$, the instantaneous received SNR $\gamma(i)$ is defined as $\gamma(i)=\bar{S}|\alpha(i)|^{2} /\left(N_{0} B\right) \quad$ and its estimate is $\hat{\gamma}(i)=\bar{S}|\hat{\alpha}(i)|^{2} /\left(N_{0} B\right)$, where $B$ denotes the received signal bandwidth. For the proposed link adaptation in Fig. 1, we assume a feedback path with negligible delay. At time $i$, the transmitter adapts to channel variation by adjusting its transmitted power $S(\hat{\gamma}(i))$ based on an SNR estimate $\hat{\gamma}(i)$ fedback from the receiver. The received SNR with adapted power $\sigma(\hat{\gamma}(i))$ is defined as $\sigma(\hat{\gamma}(i)) \triangleq \frac{S(\hat{\gamma}(i))}{\bar{S}} \hat{\gamma}(i)$. The transmitter adapts rate by adjusting its constellation size $M$ based on $\sigma(\hat{\gamma}(i))$ and received BER estimated at the receiver. An estimate of BER is obtained using the maximum a posteriori (MAP) estimation approach based on $\hat{\gamma}(i)$.

Since the channel model is assumed to be statistically stationary, the distribution of the channel gain and SNR are independent of time, and hence we shall omit the discrete time reference $i$ in the following sections.

\section{BER Estimate}

In this section, we derive the estimate of BER $p_{B}$ based on Bayesian estimation with the MAP approach [6]. In Bayesian estimation, the unknown parameter is viewed as the value of a random variable (RV) and the objective is to estimate the value of the unknown parameter in terms of the observed data of another RV. In our context, we wish to estimate $p_{B}$ in terms of the observation of $\hat{\gamma}$. In adopting the MAP approach, the aim is to find an expression for the estimate $\hat{p}_{B}$ that maximizes the posterior probability density function (PDF) $f\left(p_{B} \mid \hat{\gamma}\right)$. We consider the instantaneous BER $p_{B}$ as unknown and aim to determine its estimate $\hat{p}_{B}$ in terms of the observation of $\hat{\gamma}$. By Bayes' theorem [7], we have

$$
f\left(p_{B} \mid \hat{\gamma}\right)=\frac{f\left(\hat{\gamma} \mid p_{B}\right) f\left(p_{B}\right)}{f(\hat{\gamma})},
$$

where $f(\hat{\gamma})$ is the PDF of $\hat{\gamma}, f\left(p_{B}\right)$ is the PDF of $p_{B}$ and $f\left(\hat{\gamma} \mid p_{B}\right)$ is the conditional PDF of $\hat{\gamma}$ given $p_{B}$. From (1) and without loss of generality, we remove the normalization factor $f(\hat{\gamma})$ and express it equivalently as the MAP equation

$$
l_{M A P}\left(p_{B}\right) \triangleq f\left(p_{B}, \hat{\gamma}\right)
$$

where $f(.$,$) is the joint PDF of \hat{\gamma}$ and $p_{B}$. Finally, we obtain the $\hat{p}_{B}$ expression by solving for the root of the equation

$$
\left.\frac{\partial l_{M A P}}{\partial p_{B}}\right|_{p_{B}=\hat{p}_{B}}=0 .
$$

We will show the full derivations in the following section.

\section{A. Derivations and Results}

We consider the imperfect channel estimates scenario where $\hat{\gamma} \neq \gamma$ and $\hat{\gamma}$ is fedback to the transmitter with negligible delay. Assume the transmitter adapts its power and rate relative to a BER target (BERT) based on the channel estimate $\hat{\gamma}$ instead of the actual $\gamma$. Based on the generic approximate BER expression of a MQAM scheme [2][3], we express the instantaneous BER at the receiver as $[3,(42)]$

$$
p_{B}(\gamma, \hat{\gamma})=c_{1} \exp \left(-\frac{c_{2} \gamma}{M(\hat{\gamma})-1} \frac{S(\hat{\gamma})}{\bar{S}}\right)
$$

where $c_{1}$ and $c_{2}$ are positive real numbers [2][3]. $M($.) defines the constellation size of the MQAM scheme Assume $\sigma$ is a particular value of $\sigma(\hat{\gamma})$ at the receiver. Consequently, at the transmitter, for a required BERT, the corresponding value of $M($.$) is$

$$
M=1+\frac{c_{2} \sigma}{-\ln \left(B E R T / c_{1}\right)} .
$$

We could therefore express the BER at the receiver as a function of $\gamma$ and $\hat{\gamma}$, i.e. 


$$
p_{B}=c_{1} \exp \left(-\frac{c_{2} \sigma}{M-1} \frac{\gamma}{\hat{\gamma}}\right)
$$

In a Rayleigh flat-fading channel using the PSAM technique for channel estimation [8], the amplitudes $\alpha$ and $\hat{\alpha}$ have a bivariate Rayleigh distribution. Consequently, the equivalent joint PDF of $\gamma$, and its estimate $\hat{\gamma}$, is

$$
f(\gamma, \hat{\gamma})=\frac{1}{(1-\rho) \Gamma \hat{\Gamma}} I_{0}\left(\frac{2 \sqrt{\rho}}{1-\rho} \sqrt{\frac{\gamma \hat{\gamma}}{\Gamma \hat{\Gamma}}}\right) \exp \left(-\frac{1}{1-\rho}\left(\frac{\gamma}{\Gamma}+\frac{\hat{\gamma}}{\hat{\Gamma}}\right)\right) u(\gamma) u(\hat{\gamma})
$$

where $\Gamma=E[\gamma], \hat{\Gamma}=E[\hat{\gamma}]$ and $\rho$ is the correlation coefficient between $\gamma$ and $\hat{\gamma}$. E [.] denotes the statistical expectation operator. $I_{0}($.$) is the zero order modified Bessel function$ and $u($.$) is the unit step function. Exploiting (6) and (7), and$ through a transformation of the random variables, we obtain the joint PDF of $\hat{\gamma}$ and $p_{B}$ as

$$
f\left(p_{B}, \hat{\gamma}\right)=c\left(p_{B}\right) I_{0}\left(b\left(p_{B}\right) \hat{\gamma}\right) \exp \left(-a\left(p_{B}\right) \hat{\gamma}\right)
$$

where $\quad a\left(p_{B}\right)=\frac{1}{1-\rho}\left(\frac{-(M-1)}{\sigma c_{2} \Gamma} \ln \left(\frac{p_{B}}{c_{1}}\right)+\frac{1}{\hat{\Gamma}}\right)$,

$$
\begin{gathered}
b\left(p_{B}\right)=\frac{2 \sqrt{\rho}}{1-\rho} \sqrt{\frac{-(M-1)}{\sigma c_{2} \Gamma \hat{\Gamma}} \ln \left(\frac{p_{B}}{c_{1}}\right)}, \\
c\left(p_{B}\right)=\frac{(M-1) \hat{\gamma}}{p_{B} c_{2} \sigma(1-\rho) \Gamma \hat{\Gamma}} .
\end{gathered}
$$

We then substitute (8) into (2), and remove constant terms which yields the final form of the MAP equation

$$
l_{M A P}\left(p_{B}\right)=\frac{1}{p_{B}} I_{0}\left(b\left(p_{B}\right) \hat{\gamma}\right) \exp \left(-a\left(p_{B}\right) \hat{\gamma}\right) .
$$

To utilize (12), we based on a higher SNR and $\rho$, and find an approximate equivalent using the truncated polynomial approximation of $I_{0}(.) \exp ().[9]$,

$$
l_{M A P_{-} e q}\left(p_{B}\right)=\frac{1}{p_{B}} \frac{\exp \left(b\left(p_{B}\right) \hat{\gamma}\right)}{\sqrt{2 \pi b\left(p_{B}\right) \hat{\gamma}}} \exp \left(-a\left(p_{B}\right) \hat{\gamma}\right) .
$$

We substitute (13) into (3) and obtain an approximate expression for $\hat{p}_{B}$ in closed form

$$
\hat{p}_{B} \approx c_{1} \exp \left(\frac{\rho \frac{-\sigma c_{2}}{M-1} \frac{\Gamma}{\hat{\Gamma}}}{\left((1-\rho) \frac{\sigma c_{2}}{M-1} \frac{\Gamma}{\hat{\gamma}}-1\right)^{2}}\right)
$$

We substitute (5) into (14), and simplify the received BER estimate as a function of $\hat{\gamma}$

$$
\hat{p}_{B}(\hat{\gamma})=c_{1} \exp \left(\frac{\ln \left(B E R T / c_{1}\right) \frac{\Gamma}{\hat{\Gamma}} \rho}{\left(-\ln \left(B E R T / c_{1}\right) \frac{\Gamma}{\hat{\gamma}}(1-\rho)-1\right)^{2}}\right) .
$$

We note that (15) relates to the CSI parameters $\Gamma, \hat{\Gamma}$ and $\rho$. In general, $\Gamma$ and $\hat{\Gamma}$ define respectively the average received SNR and its estimate for any channel estimation technique over a long term channel variation, while $\rho$ defines the correlation between $\gamma$ and its estimate $\hat{\gamma}$ for any channel estimation technique over a short term variation.

\section{OPtimal Rate AND Power AdAPTATION}

At the transmitter, we adjust constellation size $M$ based on estimates $\hat{p}_{B}$ and $\hat{\gamma}$. We could therefore express the adaptive $M$ as a function of $\hat{\gamma}$

$$
M(\hat{\gamma})=1+\frac{c_{2} \sigma(\hat{\gamma})}{-\ln \left(\hat{p}_{B}(\hat{\gamma}) / c_{1}\right)} .
$$

Substituting $\hat{p}_{B}(\hat{\gamma})$ of (15) into (16), the final form of $M($.) simplifies to

$M(\hat{\gamma})=1+\frac{K}{p} \frac{\hat{\Gamma}}{\Gamma}\left\{-\ln \left(B E R T / c_{1}\right)(1-\rho) \frac{\Gamma}{\hat{\gamma}}-1\right\}^{2} \frac{S(\hat{\gamma})}{\bar{S}} \hat{\gamma}$, 
where $K=\frac{c_{2}}{-\ln \left(B E R T / c_{1}\right)}$. The spectral efficiency of a MQAM scheme can be expressed as its average data rate per unit bandwidth $R / B$. With data sent at $k(\hat{\gamma})=\log _{2}(M(\hat{\gamma}))$ bits/symbol, the instantaneous data rate is $k(\hat{\gamma}) / T_{s}$ (bits/sec), where $T_{s}$ is the symbol time. Assuming Nyquist data pulses of duration $T_{s}=1 / B$, the spectral efficiency (bits/sec/Hz) for continuous rate can be given by

$$
\frac{R}{B}=\int_{\hat{\gamma}} k(\hat{\gamma}) f(\hat{\gamma}) d \hat{\gamma}
$$

We derive the optimal rate and power adaptation by maximizing spectral efficiency (18) subject to the average power constraint

$$
E[S]=\int_{\hat{\gamma}} S(\hat{\gamma}) f(\hat{\gamma}) d \hat{\gamma} \leq \bar{S}
$$

where $f(\hat{\gamma})$ denotes the PDF of $\hat{\gamma}$. To solve the constrained optimization problem, we form the Lagrangian equation

$$
F(S(\hat{\gamma}))=\int_{\hat{\gamma}} k(\hat{\gamma}) f(\hat{\gamma}) d \hat{\gamma}+\lambda\left[\int_{\hat{\gamma}} S(\hat{\gamma}) f(\hat{\gamma}) d \hat{\gamma}-\bar{S}\right]
$$

where $\lambda$ denotes the Lagrange multiplier. We solve $\partial F / \partial S(\hat{\gamma})=0$ for $S(\hat{\gamma})$ and obtain the optimal power adaptation

$$
\frac{S(\hat{\gamma})}{\bar{S}}_{o p}=\left\{\begin{array}{cc}
U-\frac{\frac{\Gamma}{\hat{\Gamma}} \rho}{K \hat{\gamma}\left\{\frac{c_{2}}{K} \frac{\Gamma}{\hat{\gamma}}(1-\rho)-1\right\}^{2}}, k(\hat{\gamma}) \geq 0, S(\hat{\gamma})>0, \\
0, & \text { otherwise, }
\end{array}\right.
$$

where $U=\frac{1}{-\lambda \bar{S} \ln (2)}$ is a constant value found through numerical search such that the power constraint (19) is satisfied. We substitute (21) into (17) and obtain the optimal rate adaptation as

$$
k_{o p}(\hat{\gamma})=\left\{\begin{array}{cc}
\log _{2}\left(\begin{array}{c}
\left.\hat{\gamma} U \frac{c_{2}}{K} \frac{\Gamma}{\hat{\gamma}}(1-\rho)-1\right\}^{2} \\
\rho \frac{\Gamma}{\hat{\Gamma}}
\end{array}\right), k(\hat{\gamma}) \geq 0, S(\hat{\gamma})>0, \\
0, & \text { otherwise. }
\end{array}\right.
$$

Finally, by substituting (22) into (18), the maximum spectral efficiency can be obtained.

\section{Numerical REsults}

In this numerical evaluation, we focus on the performance of the system over short-term variations modelled by a Rayleigh distribution with $f(\hat{\gamma})=\frac{1}{\hat{\Gamma}} \exp (-\hat{\gamma} / \hat{\Gamma})$. We assume $\hat{\Gamma}=\Gamma$, where a perfect channel estimate is possible over a long-term channel and represent the short-term imperfect channel estimation by $\rho<1$. In the case of such partial knowledge of CSI, we use power and rate adaptations of (21) and (22) respectively in (18) to compute for maximum spectral efficiency.

For comparison to a similar system of [3], which corresponds to a variable rate and power MQAM system optimized by assuming perfect knowledge of CSI, we let $U=1 / \gamma_{0}, c_{1}=0.2$ and $c_{2}=1.5$. Note $\gamma_{0} / K$ is defined as the optimized cutoff fade depth. In fact, when $\rho=1$, (21) and (22) simplify to the same optimal power and rate adaptations of [3]. We show in Fig. 2 the spectral efficiency assuming a continuous MQAM with a BER target of $10^{-3}$. The spectral efficiency curve illustrates the same performance as achieved in [3].

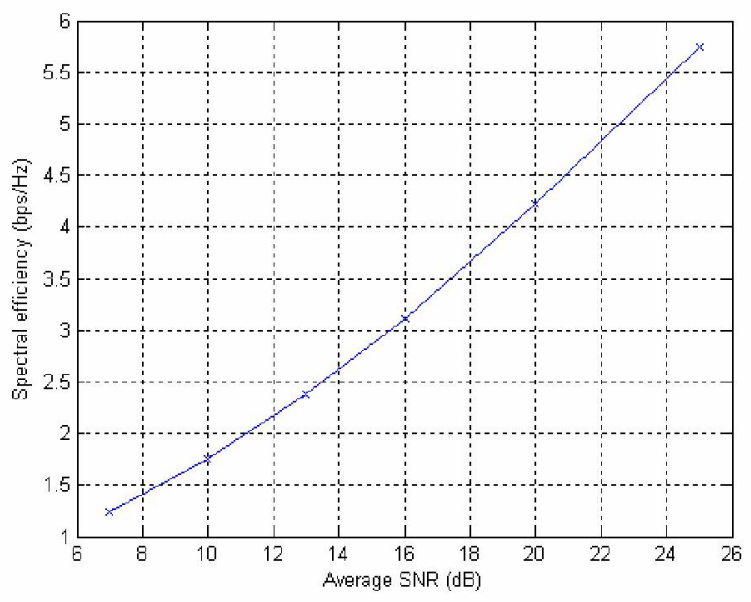

Figure 2. Spectral efficiency as a function of average SNR with BER target $10^{-3}$ and perfect CSI. 


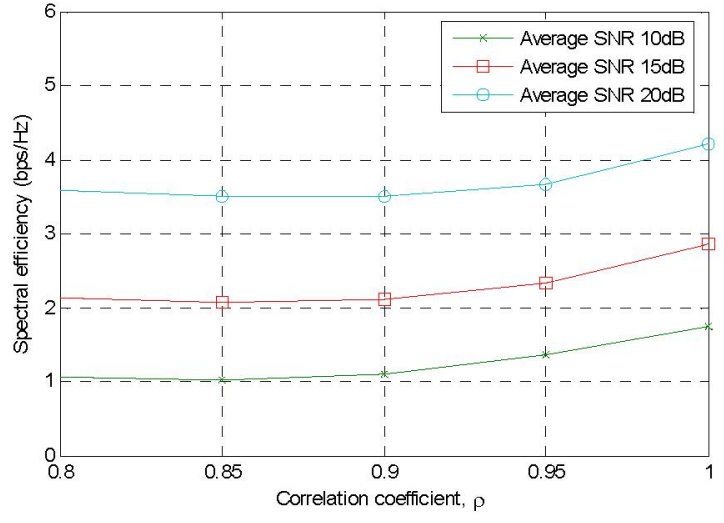

Figure 3. Spectral efficiency as a function of correlation coefficient for three settings of average SNR.

To study the impact of the variation of spectral efficiency and average power over $\rho$, we compute numerical results for spectral efficiency as a function of $\rho$ and the normalized average power as a function of $\rho$ using

$$
E\left[S_{n o r m}\right]=\int_{\hat{\gamma}} \frac{S(\hat{\gamma})}{\bar{S}} f(\hat{\gamma}) d \hat{\gamma}
$$

where $S(\hat{\gamma}) / \bar{S}$ is from (21). We show in Fig. 3 and Fig. 4 the results of spectral efficiency and average power over $\rho$ for a set of three average SNR values: $\Gamma=10 \mathrm{~dB}, 15 \mathrm{~dB}$ and $20 \mathrm{~dB}$. The two graphs depict that optimal spectral efficiency is achieved with normalized average power of $0 \mathrm{~dB}$ when $\rho=1$; and that spectral efficiency is reduced and average power varies when $\rho<1$. Furthermore the two graphs illustrate several interesting points in terms of the variation of spectral efficiency and average power over $\rho$ and $\Gamma$ parameters. First, the rate of change of spectral efficiency is identical for all $\Gamma$ values. The spectral efficiency decreases by approximately $0.5 \mathrm{bit} / \mathrm{sec} / \mathrm{Hz}$ at $0.95<\rho<1$ and is almost insignificant at $0.8<\rho<0.9$. In contrast, the rate of change of average power varies across the values of $\Gamma$ and $\rho$. To illustrate with numeric data, Fig. 4 shows that for $\rho<0.9$ the average power for $\Gamma=10 \mathrm{~dB}$ increases at a rate of $1 \mathrm{~dB}$ per unit of $\rho$ as compared to a rate of $0.3 \mathrm{~dB}$ per unit of $\rho$ when $\Gamma=20 \mathrm{~dB}$.

\section{CONCLUSIONS}

We have obtained a BER estimator for a variable rate and variable power MQAM system over a Rayleigh flat-fading channel using maximum a posteriori approach. We have also derived the rate and power adaptation based on the resulting BER estimate and the estimate of SNR. We verified that in perfect CSI, our rate and power adaptation agree with that of the adaptive rate and power MQAM system of [3], where the

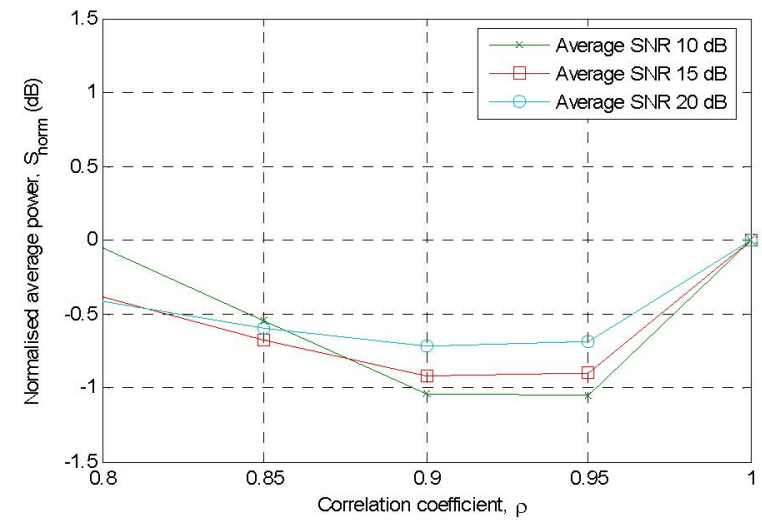

Figure 4. Normalized average power (dB) as a function of correlation coefficient for three settings of average SNR.

corresponding rate and power derivations are valid only for perfect CSI analysis. In contrast, our rate and power derivations are valid for imperfect CSI analysis. For instance through numerical evaluation, we show the impact of imperfect channel estimate on spectral efficiency and average power over a short-term channel variation. The numerical results show that for $\rho<1$, the rate of change of spectral efficiency is relatively constant for all average SNR. In contract, the rate of change of average power varies over the average SNR.

\section{ACKNOWLEDGEMENT}

The authors are grateful to Dr. Ali Olfat for his suggestions in the initial stage of this research work.

\section{REFERENCES}

[1] A. J. Goldsmith and P. P. Varaiya, "Capacity of fading channels with channel side information," IEEE Trans. Info. Theory, vol 43, pp. 1986 - 1992, Nov 1997.

[2] S.T. Chung and A. J. Goldsmith, "Degrees of freedom in adaptive modulation: A unified view," IEEE Trans. Commun., vol 49, pp. 1561-1571, Sept. 2001

[3] A. J. Goldsmith and S. Chua, "Variable-rate variable-power MQAM for fading channels," IEEE Trans. Commun., vol 45, pp.1218 -1230, Oct 1997.

[4] J. F. Paris, M. C. Aguayo-Torres, and J. T. Entrambasaguas, "Impact of channel estimation error on adaptive modulation performance in flat fading", IEEE Trans. Commun., vol 52, pp.716 -720, May 2004.

[5] J. K. Cavers, "An analysis of pilot symbol assisted modulation for rayleigh fading channels," IEEE Trans. Veh. Technol., vol 40, pp. 686 693, Nov. 1991.

[6] H. L. Van Trees, Detection, Estimation, and Modulation Theory, Part I, John Wiley and Sons, 2001.

[7] A. Papoulis, Probability, Random and Stochastic Process, McGrawHill, 1984.

[8] X. Tang, M.-S. Alouini, and A.J. Goldsmith, "Effect of channel estimation error on M-QAM BER performance in rayleigh fading," IEEE Trans. Commun., vol 47, pp.1856 -1864, Dec 1999.

[9] M. Abramowitz and I. A. Stegun, Handbook of Mathematical Functions with Formulas, Graphs, and Mathematical Tables, National Bureau of Standards Applied Mathematics Series - 55, U.S Government Printing Office, 1972. 